\section{Tratamiento odontológico de un paciente con la enfermedad de Riga-Fede. Reporte de caso clínico}

\section{Dental treatment of a patient with Riga- Fede disease. Clinical case report}

\begin{abstract}
Resumen
La enfermedad de Riga-Fede es un trastorno benigno poco común, se caracteriza por una ulceración reactiva de la mucosa oral, que surge de lesiones traumáticas repetitivas. El trauma constante puede provocar una ulceración lo suficientemente grande como para interferir en la adecuada succión y alimentación, lo que provocaría un riesgo de deficiencias nutricionales, deshidratación e infecciones. Tenemos como ayuda diagnostica, la radiografía intraoral, que en lactantes es tomada con la técnica de Mannkopf. Con respecto al tratamiento de esta lesión hay variada información en la literatura y ha cambiado a lo largo de los ańos, existen tratamientos conservadores como radicales. Se presenta el caso clínico de un bebe de sexo masculino de 7 meses, el cual presenta una ulcera en la cara ventral de lengua, con bordes definidos, de aproximadamente $3,5 \mathrm{~cm} \times 2,5 \mathrm{~cm}$ de diámetro, de color rojizo con áreas blanquecinas, dolorosa a la palpación, cursa con fiebre y con problemas de alimentación. Ingresa por infectología al Instituto Nacional de Salud del Nińo, donde se le realizan múltiples exámenes auxiliares, sin poder hallar el diagnóstico, es referido al Servicio de Cirugía Buco Maxilofacial, donde es diagnosticado y tratado con éxito.
\end{abstract}

Palabras clave: Diente; Odontología pediátrica; Úlcera oral.

\section{Caso Clínico}

Evelyn del Rosario Munayco Pantoja 1,a, Gilmer Torres Ramos ${ }^{1, b}$, Olinda Huapaya Paricoto ${ }^{1, c}$, Sylvia Antonieta Chein Villacampa ${ }^{1, d}$

1 Universidad Nacional Mayor de San Marcos, Facultad de Odontología. Lima, Perú.

${ }^{a}$ Especialista en Odontopediatria.

${ }^{\mathrm{b}}$ Doctor en Estomatología

c Magister en Estomatología

${ }^{d}$ Especialista en Salud Pública Estomatológica

Correspondencia:

Evelyn del Rosario Munayco Pantoja

Correo electrónico:

evelyn.munayco@unmsm.edu.pe

Av. Venezuela 1288 Breña, Lima, Perú.

Coautores:

Gilmer Torres Ramos

gtorresr@unmsm.edu.pe

Olinda Huapaya Paricoto

dhuapayap@unmsm.edu.pe

Sylvia Antonieta Chein Villacampa

scheinv@unmsm.edu.pe

Conflicto de intereses: Los autores declaran no tener conflictos de interés.

Fuente de financiamiento: Autofinanciado

Fecha de recepción: 09/03/18

Fecha de aceptación: 15/05/18

\begin{abstract}
Riga-Fede disease is a rare, benign disorder characterized by a reactive ulceration of the oral mucosa, which arises from repetitive traumatic injuries. The constant trauma can cause an ulceration large enough or annoying enough to interfere with proper suction and feeding, which would cause a risk of nutritional deficiencies, dehydration and infections. We have as diagnostic aid, the intraoral radiography, which in infants is taken with the Mannkopf technique. With respect to the treatment of this lesion there is varied information in the literature and it has changed over the years, there are conservative treatments as radicals. We present the clinical case of 7 -month-old male infant, who presents with an ulcer on the ventral side of the tongue, with defined edges, approximately 3.5 $\mathrm{cm} \times 2.5 \mathrm{~cm}$ in diameter, colored reddish with whitish areas, painful on palpation, with fever and eating problems. He was admitted to the National Institute of Child Health by infectology, where multiple auxiliary examinations were performed, unable to find diagnosis, he was referred to the service of BucoMaxiloFacial Surgery, where his illness was diagnosed and successfully treated.
\end{abstract}

Keywords: Oral ulcer; Pediatric dentistry; Tooth.

(c) Los autores. Este artículo es publicado por la revista Odontología Sanmarquina de la Facultad de Odontología, Universidad Nacional Mayor de San Marcos. Este es un artículo de acceso abierto, distribuido bajo los términos de la licencia Creative Commons Atribucion - No Comercia_Compartir Igual 4.0 Internacional. (http://creativecommons.org/licenses/by-nc-sa/4.0/) que permite el uso no comercial, distribución y reproducción en cualquier medio, siempre que la obra original sea debidamente citada. 


\section{Introducción}

La enfermedad de Riga-Fede es un trastorno benigno poco común de la mucosa oral, se caracteriza por una ulceración reactiva de la mucosa, que surge de lesiones traumáticas repetitivas, generalmente causado por los bordes incisales cortantes de los incisivos inferiores ${ }^{1}$. La prevalencia de aparición de estas lesiones está asociada en un 6 a $10 \%$ a los dientes natales o neonatales ${ }^{2}$. Los dientes natales aparecen desde el nacimiento y los neonatales, durante los primeros treinta días, según la clasificación de Massler y Savara ${ }^{3}$. Además, esta ulceración puede aparecer en lactantes en los que el trauma crónico de los incisivos temporales, durante el amamantamiento y el proceso instintivo de succión, origina una masa exofítica ulcerada y granulomatosa cubierta por una pseudomembrana amarillenta ${ }^{4}$. Clínicamente, en estadios iniciales, aparece como una úlcera localizada en la lengua ( $60 \%$ de las lesiones), aunque también aparece en otras áreas como labio, paladar, encía, mucosa vestibular $y$ piso de la boca ${ }^{5}$. Ambos sexos son igualmente afectados ${ }^{6}$. El trauma constante puede provocar una ulceración lo suficientemente grande como para interferir en la adecuada succión y alimentación, lo que provocaría un riesgo de deficiencias nutricionales, deshidratación e infecciones ${ }^{7}$.

Este tipo de úlcera fue descrita por primera vez por Caradelli en 1875 y seis años más tarde por Antonio Riga. Posteriormente, en 1890 Francesco Fede definió la lesión histológicamente y reportó 20 casos; desde entonces se conoce con el nombre de enfermedad de Riga-Fede, que denomina a la lesión lingual ulcerativa que aparece en bebes poco después de la erupción de los incisivos inferiores. Pero varios términos son utilizados para denotar estas lesiones y al parecer no hay un consenso sobre la nomenclatura ${ }^{8}$. Se le conoce con las siguientes denominaciones "úlcera sublingual”, "granuloma sublingual", "lesión reparativa de la lengua" 7 " "granuloma ulcerativo traumático con estroma eosinófilo", termino sugerido por Elzay ${ }^{9}$ para describir el traumatismo ulcerativo sublingual en las lesiones observadas en niños y adultos; y "ulceración sublingual traumática neonatal", término usado por Goho ${ }^{10}$ para denotar específicamente las lesiones sublinguales que ocurren en los recién nacidos debido a la lesión traumática de la lengua por los dientes natales o neonatales.

Las úlceras en la lengua pueden tener otras etiologías que incluyen alergias, infecciones bacterianas, trastornos inmunológicos y condiciones metastásicas. Algunos pacientes con síndromes congénitos y alteraciones neurológicas presentan automutilación oral (comportamiento auto-agresivo) como el Síndrome de Riley-Day ', Síndrome de Lesch-Nyhan, parálisis cerebral, Síndrome neuro-hematológico, etc ${ }^{11}$. Esto debe ser diferenciado de otras patologías como candidiasis ulcerativa, agranulocitosis, sífilis primaria, sarcoma, linfoma y tuberculosis ${ }^{6,12}$. Por ello, es importante la historia clínica (antecedentes familiares), características clínicas y datos de laboratorio ${ }^{11}$.
Una ayuda diagnóstica es la radiografía dental, la cual se emplea para determinar si los dientes son parte de la dentición primaria, supernumerarios, dientes natales, neonatales y el estado de formación de la raíz ${ }^{3}$. Existe una técnica radiográfica para recién nacidos y lactantes, citado por Hardnt ${ }^{13}$, denominada técnica de Mannkopf, la cual consiste en posicionar la película en posición oclusal contra el rodete gingival.

Con respecto al tratamiento de esta lesión la información ha cambiado a lo largo de los años ${ }^{11}$. Existen tratamientos conservadores como modificar el comportamiento de alimentación con el uso de un dispositivo (cuchara) ${ }^{8}$, destetar al infante, tratar solo los síntomas, desgastar los bordes incisales ${ }^{6}$, colocar resina compuesta o no realizar ningún tratamiento y solo controles periódicos. Con respecto a la colocación de la resina compuesta, esta depende de un adecuado control de la humedad, porque de lo contrario la restauración fracasaría y podría ser tragada ${ }^{11}$. Existen también tratamientos radicales como extraer los incisivos inferiores, tratar la lesión con radiación, aplicar nitrógeno líquido y extirpar la lesión ${ }^{7}$.

\section{Reporte del caso}

Paciente de sexo masculino de 7 meses de edad, natural y procedente de Chosica, Lima, Perú. No presenta enfermedades sistémicas, no hay antecedentes familiares de trastornos del desarrollo ni síndromes congénitos. Madre refiere que aparece lesión ulcerada después de la erupción de los incisivos inferiores, hace 1 mes, inicialmente con un tamańo de $1,5 \mathrm{~cm}$ x $1,5 \mathrm{~cm}$, ubicado en la región sublingual, dolorosa, con restos blanquecinos y superficie sangrante al tacto, cursa con fiebre que alcanza los $38,5-39,5^{\circ} \mathrm{C}$ con presentación hasta cada 3 o 4 horas. En el hospital de Vitarte le prescriben enjuagues bucales y antipiréticos; posteriormente solicitan exámenes basales y radiografía de tórax con resultados dentro de lo normal. En los siguientes días, la herida aumenta de tamańo progresivamente, se torna bien delimitada, persiste sangrado y dolor. Es referido al Instituto Nacional de Salud del Niño, donde ingresa por emergencia al área de Infectología, presentaba fiebre persistente y sin poder alimentarse. Es hospitalizado durante 11 días, donde le realizan múltiples exámenes auxiliares e interconsultas. El resultado de los análisis solicitados fue negativo, no encontraron nada anormal ni en heces, orina, sangre, médula, biopsia, etc. Al estar la lesión localizada en cavidad oral, y al no hallar la causa probable, solicitan una interconsulta con el Servicio de Cirugía Buco Maxilofacial. La fiebre había sido controlada debido a la administración de paracetamol vía oral.

Al examen clínico extraoral presentaba una facie dolorosa. Presenta tres lesiones ulcerosas de aproximadamente $1 \mathrm{~cm}$ x $0,5 \mathrm{~cm}$ en cara dorsal de la mano derecha (Figura 1).

Al examen clínico intraoral presenta lesión ulcerosa granulomatosa en cara ventral de lengua, con bordes definidos, de aproximadamente $3,5 \mathrm{~cm}$ x 2,5 cm de diámetro, de color rojizo con áreas blanquecinas (Figura 2). Presenta piezas dentarias $71,72,81$ con bordes cortantes y con movilidad grado I. 
En la radiografía se observa que existe una fusión de las piezas 81,82 . Las piezas $71,72,81,82$ pertenecen a la serie normal de erupción (Figura 3). Los análisis de sangre descartaban algún problema de coagulación.

Se diagnosticó enfermedad de Riga-Fede y se descartó la asociación de la úlcera con enfermedades del trastorno del desarrollo o síndromes congénitos, debido a que no hay antecedentes en el historial familiar.

El resultado del examen anatomopatológico de la muestra obtenida en sala de operaciones por el Servicio de Cabeza y Cuello fue: tejido muscular ulcerado, con áreas de tejido de granulación, vasculitis y denso infiltrado in-

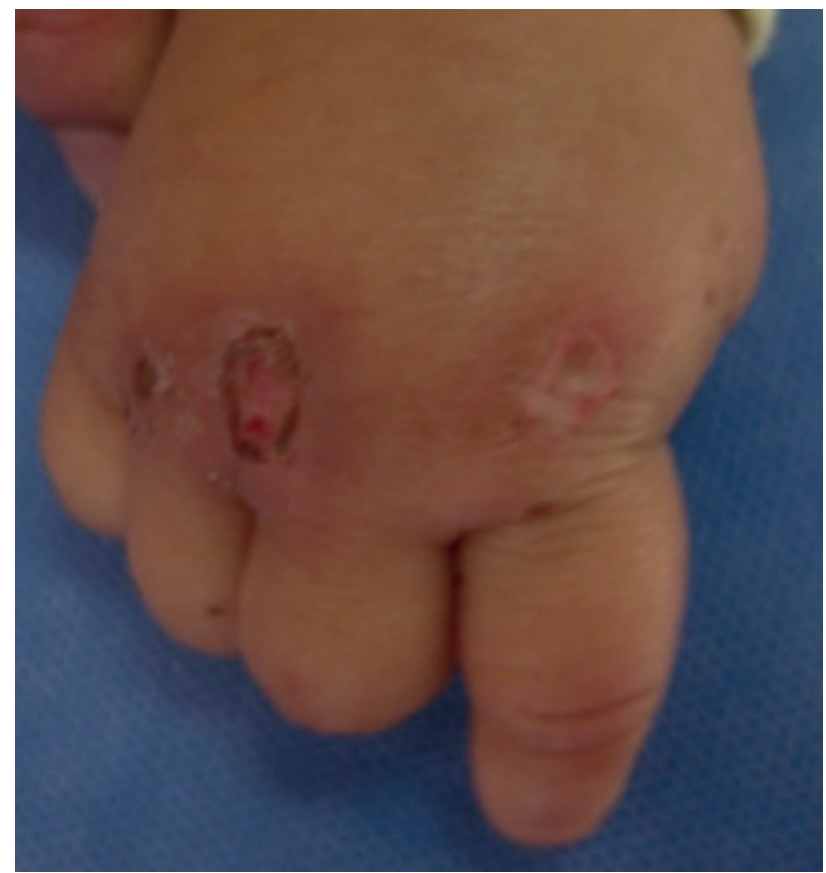

Figura 1. Examen clínico extraoral donde se observa tres lesiones ulcerosas de aproximadamente $1 \mathrm{~cm} \times 0,5 \mathrm{~cm}$ en cara dorsal de la mano derecha flamatorio agudo, fibrino leucocitario con microabcesos, no se encontraron células neoplásicas.

Tratamiento. Para decidir qué tratamiento seguir se tomó en cuenta: las faltas nutricionales del paciente, la recurrencia de fiebre, tiempo hospitalizado, tamaño de la lesión, múltiples análisis auxiliares a los que ha estado expuesto y sobre todo preocupación de la madre. Se decidió por un tratamiento no conservador. Se explicó a los padres los riesgos y beneficios del tratamiento, previa firma del consentimiento informado. Se aplicó lidocaína $2 \%$ con epinefrina 1:80 000 con una aguja $31 \mathrm{G}(0,25$ $\mathrm{mm} \times 12 \mathrm{~mm}$ ), se procedió a realizar las exodoncias de las piezas 71, 72, 81, 82 (Figura 4). Se indicó la higiene

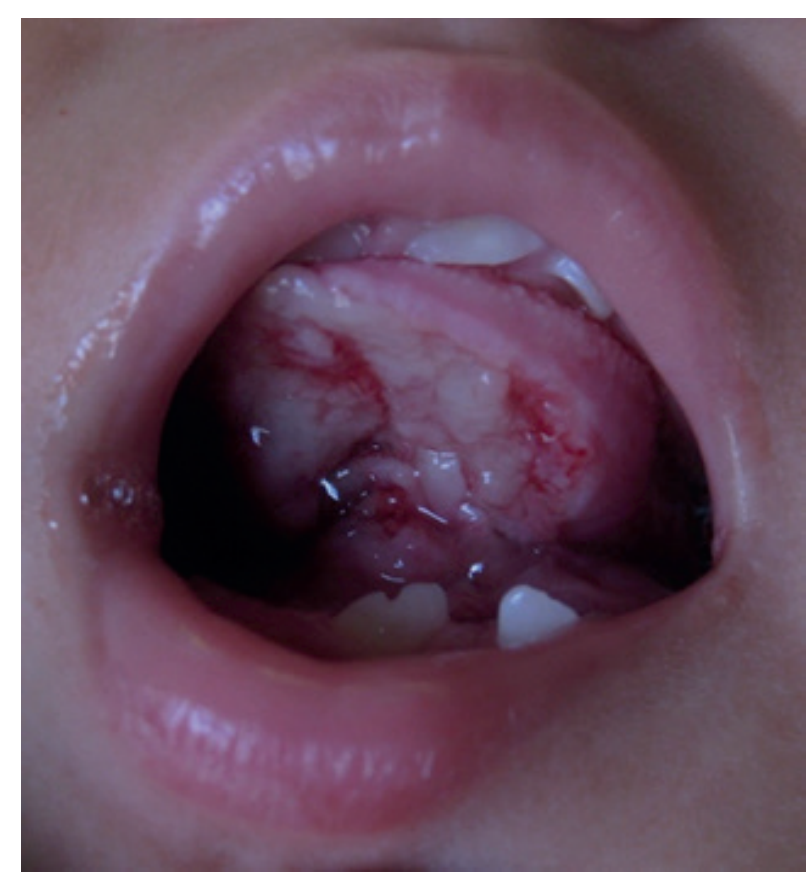

Figura 2. Examen clínico intraoral donde se observa lesión ulcerosa granulomatosa en cara ventral de lengua, con bordes definidos, de aproximadamente $3,5 \mathrm{~cm} \times 2,5 \mathrm{~cm}$ de diámetro, de color rojizo con áreas blanquecinas

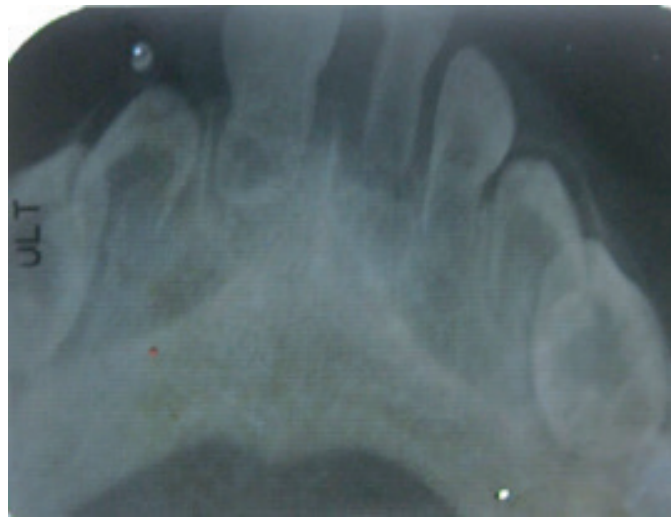

Figura 3. Radiografía intraoral tomada según la técnica de Mannkopf donde se observa que existe una fusión de las piezas 81,82 . Todas pertenecen a la serie normal de erupción

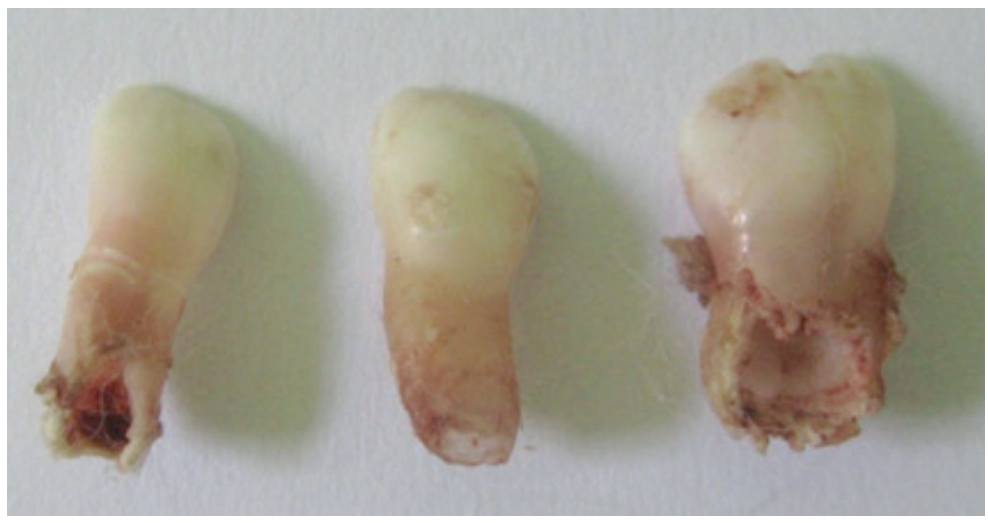

Figura 4. Se observa piezas $71,72,81,82$, con bordes filosos, recién extraídas 
con clorhexidina $0,12 \%$ dos veces al día y medicación vía oral con Ibuprofeno $100 \mathrm{mg} / 5 \mathrm{ml}$ en suspensión, una cucharadita cada 8 horas por 2 días.

Se realizó el primer control a la semana siguiente de la cirugía, donde se encontró la mucosa cicatrizando con normalidad, madre refiere que el paciente se puede alimentar adecuadamente (Figura 5). El segundo control se realizó al mes de la cirugía, donde se puede observar que la herida estaba remitiendo (Figura 6). No se encontraron signos de recurrencia después de 3 meses de controles.

\section{Discusión}

Convencionalmente el término de Riga-Fede se utiliza para denotar la ulceración de la superficie ventral de la lengua causada por movimientos repetitivos protrusivos y retrusivos de los incisivos inferiores ${ }^{14}$. En este reporte de caso, la úlcera de Riga-Fede fue provocada por dientes de la dentición normal, pero la literatura refiere más casos de esta lesión relacionado con los dientes natales o neonatales. Estos dientes son usualmente ubicados en la región de los incisivos inferiores, aparecen en par en un $61 \%$ de los casos, de estos el $95 \%$ corresponden a

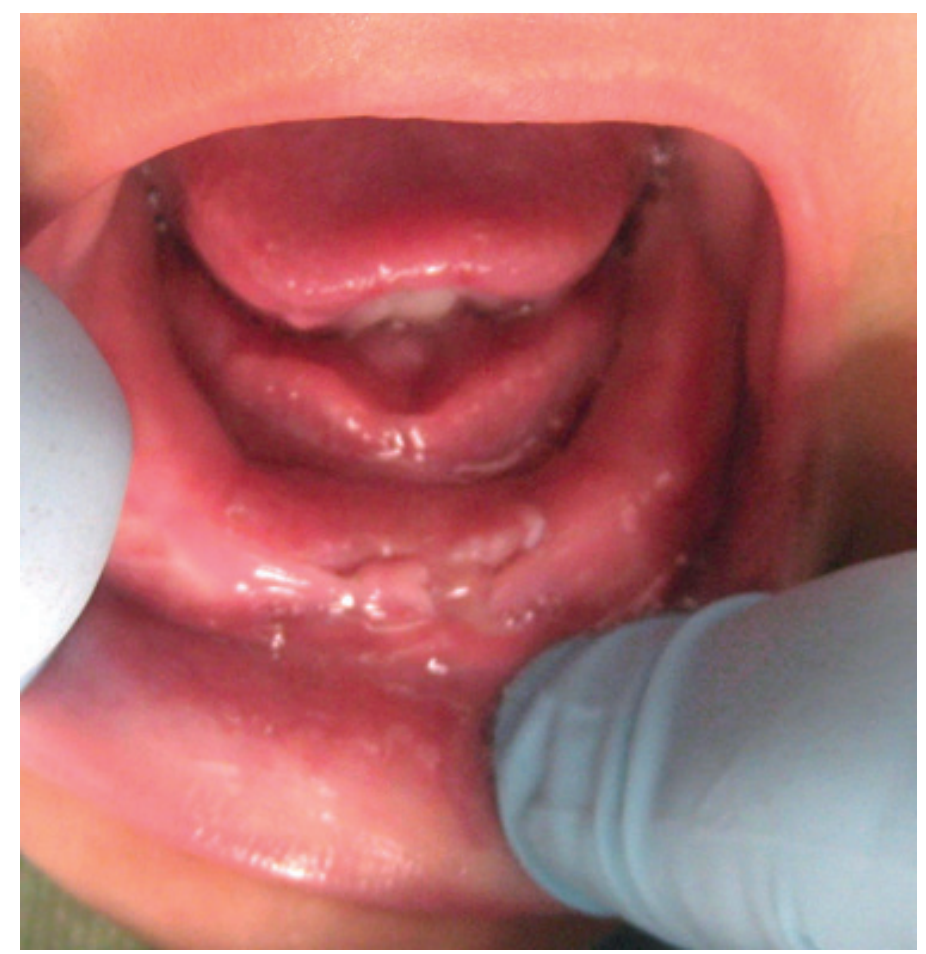

Figura 5. Primer control del paciente una semana después de la extracción, se observa la mucosa lingual en proceso de cicatrización

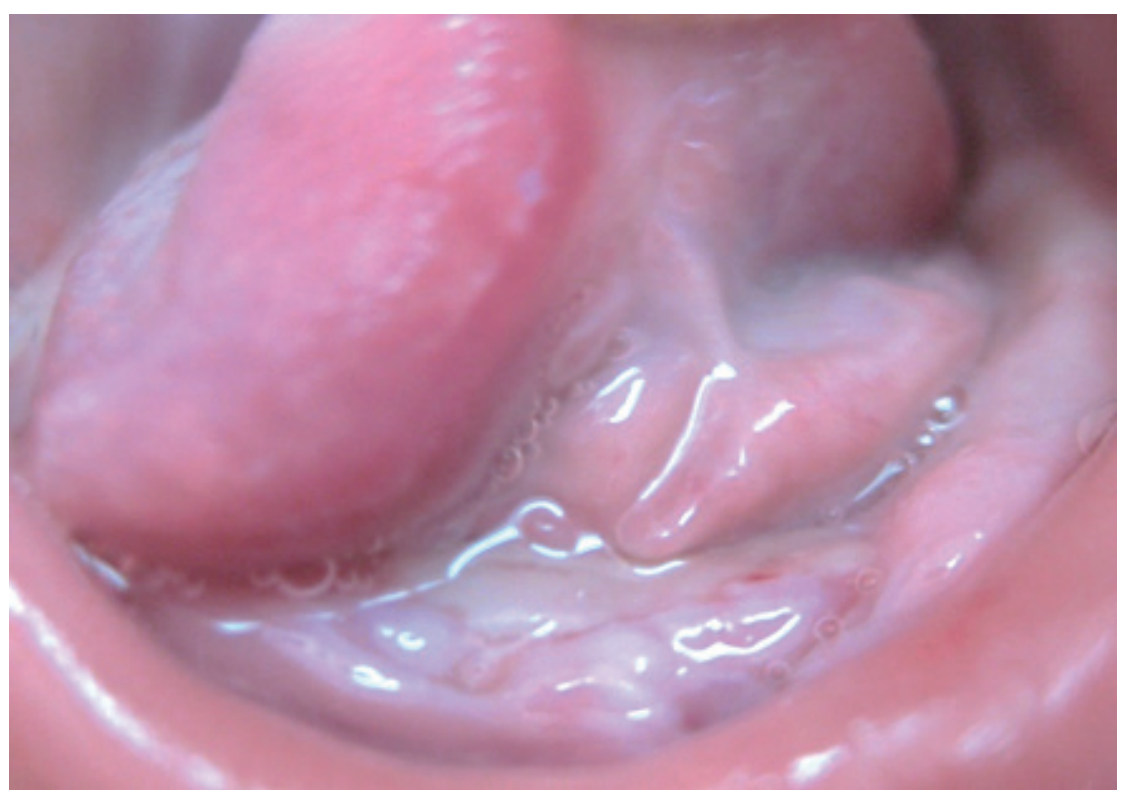

Figura 6. Segundo control del paciente un mes después de la extracción, se observa la mucosa sana 
los dientes de la dentición primaria mientras que el 5\% son supernumerarios ${ }^{15}$. Rao et al ${ }^{16}$ refiere que $90-99 \%$ de los dientes natales-neonatales son dientes de la dentición normal y 1-10\% son supernumerarios.

En este estudio, la biopsia de la lesión realizada en sala de operaciones fue indicada por el Servicio de Cabeza y Cuello, con respecto a este procedimiento no hay un consenso en la literatura. Sung ${ }^{17}$ no realizó la biopsia por considerar la edad del bebe (8 meses) y porque la evidencia clínica fue suficiente para el diagnóstico. Sin embargo, si la lesión persiste después del tratamiento, se debe realizar una biopsia por escisión bajo anestesia general ${ }^{7,18}$. Según la literatura, el resultado de la biopsia de esta lesión es definida como proliferaciones histiocíticas y fibroblásticas que se infiltran entre estriados haces musculares con números variables de eosinófilos, neutrófilos y células plasmáticas resultado similar a lo hallado en este estudio ${ }^{11,20}$.

Para la mayoría de las lesiones traumáticas, el objetivo principal es eliminar la fuente del trauma. Si es un diente de la dentición normal, este debe conservarse y mantenerse en condiciones saludables en la boca del bebé usando todos los recursos clínicos posibles y así promover la curación de la lesión ${ }^{15}$. En general, los tratamientos conservadores son de primera elección, excepto en los casos donde el niño presenta desnutrición y/o deshidratación debido a que tienen un valor limitado ${ }^{7,14}$. Extraer los incisivos inferiores puede ser el tratamiento de elección ${ }^{12}$. Sin embargo, si las lesiones persisten, se debe realizar la biopsia y otras pruebas de diagnóstico ${ }^{17}$. En este caso, se eligió como tratamiento la extracción de las piezas deciduas, para que haya una resolución rápida de la lesión y por la insuficiente ingesta de nutrientes; similar tratamiento fue realizado por Domingues-Cruz y Herrera ${ }^{1}$ que en un inicio intentaron realizar un tratamiento conservador pero la lesión persistió, por lo que al final optaron por un tratamiento con extracciones. Otro caso registrado es el de Yuvaraj $e t a l^{8}$, el cual realizó un tratamiento conservador a pedido de los padres, en dos semanas la lesión persistió y decidió realizar la exodoncia en sala de operaciones, también realizó un legrado suave del alveolo para evitar el desarrollo de células de la vaina epitelial de Hertwigs. Este autor también presentó un esquema de tratamiento para la Enfermedad de Riga-Fede (Figura 7).

En el caso de que los dientes sean supernumerarios, estos deben ser extraídos ${ }^{15}$. Se debe evitar la extracción hasta el décimo día de vida para prevenir la hemorragia, este período de espera es debido a que la flora comensal del intestino tiene que establecerse y producir vitamina $\mathrm{K}$, que es esencial para la producción de protrombina en el hígado. Si no es posible esperar, entonces es aconsejable evaluar la necesidad de la administración de vitamina $\mathrm{K}$ (0,5-1,0 mg intramuscular) con un pediatra antes de la extracción, teniendo en cuenta el estado general de salud del bebé ${ }^{15}$. Con respecto a los anestésicos locales usados para el procedimiento, existe referencia en la literatura del uso de la benzocaína, como anestesia tópica, en ex-

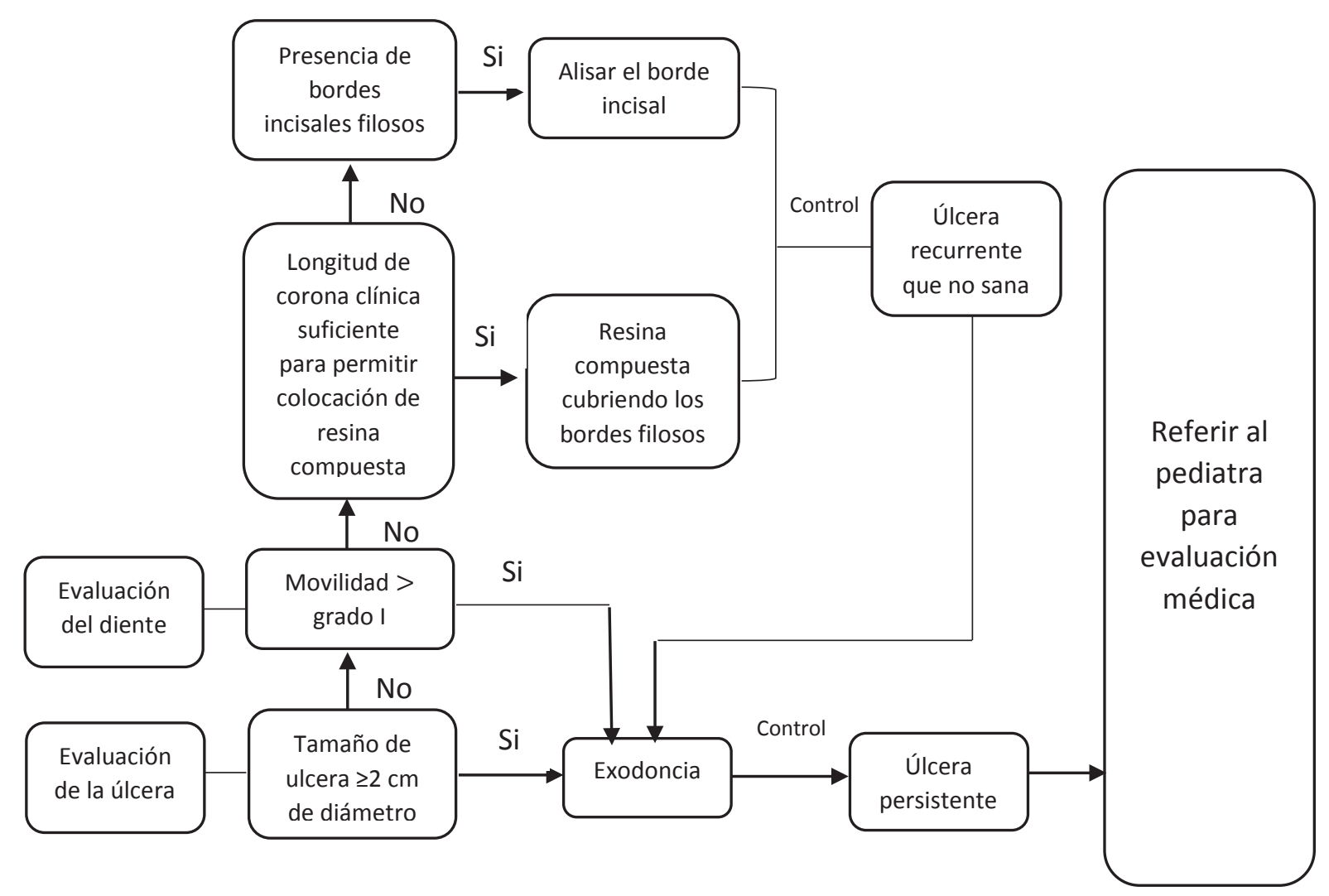

Figura 7. Esquema de tratamiento de la enfermedad de Riga-Fede (modificado de Yuvaraj ${ }^{8}$ ) 
tracciones de dientes neonatales con movilidad II ${ }^{21}$. En este caso, solo se usó la lidocaína, para evitar una condición médica llamada metahemoglobinemia ${ }^{22}$. Según el estudio de Guay ${ }^{23}$, el cual hizo una revisión de 242 casos de metahemoglobinemia por anestésicos locales, la benzocaína estaba relacionada en $66 \%$ de los casos mientras que la lidocaína, solo en el 5\%. También Veltri y Rudnick ${ }^{24}$ clasificaron a la benzocaína y prilocaína como un agente de alto riesgo, mientras que a la lidocaína como moderado riesgo de producir metahemoglobinemia. Para Schroeder $e a^{2} l^{25}$ la prilocaína es el anestésico tópico más probable en causar esta condición médica.

Las consecuencias de la decisión de extraer un diente deciduo a edad temprana son: pérdida de espacio, colapso del arco mandibular en desarrollo y la maloclusión consiguiente en la dentición permanente ${ }^{15}$. Ziad ${ }^{11}$ observó también inclinación mesial de los dientes adyacentes y estrechez del espacio, por lo que se recomienda realizar controles periódicos, a fin de evitar estas complicaciones. Por otro lado, el tiempo en que la lesión remite o es resuelta es muy variable y no depende del tipo de tratamiento a escoger, en este caso se optó por un tratamiento radical en donde la lesión se resolvió en un mes, para Domingues-Cruz y Herrera ${ }^{1}$ fueron 3 semanas, Costacurta et al ${ }^{21} ; 4$ semanas, Yuvaraj et al ${ }^{8} ; 1$ mes, comparado con otros autores que realizaron tratamientos conservadores como Sule y Bilal ${ }^{6}$ en 2 semanas, Ziad ${ }^{14}$ en 25 días y Slayton ${ }^{7}$ en 1 mes.

Se concluye que es necesario realizar un diagnóstico diferencial eficaz y un tratamiento oportuno, esto para evitar la deshidratación, ingesta inadecuada de nutrientes y otras secuelas médicas. La decisión sobre qué tratamiento elegir debe ser evaluado en cada caso, teniendo en cuenta el conocimiento científico, sentido común clínico y opinión de los padres.

\section{Referencias bibliográficas}

1. Domingues-Cruz J, Herrera A. Riga-Fede disease associated with postanoxic encephalopathy and trisomy 21: a proposed classification. Pediatr Dermatol. 2007;24(6):663-665.

2. To E. A study of natal teeth in Hong Kong Chinese. Int J Paediatr Dent. 1991;1(7):73-76.

3. Kana A, Markou I, Arhakis A, Kotsanos N. Natal and neonatal teeth: a systematic review of prevalence and management. European Journal of Paediatric Dentistry. 2013;14(1):27-32.

4. Boj J, Catalá M, García-Ballesta C, Mendoza A, Planells P. Odontopediatria. La evolución del niño al adulto joven. $1^{\text {a }}$ ed. Madrid: Ripano S.A; 2011.

5. Joseph B, BairavaSundaram D. Oral traumatic granuloma: Report of a case and review of literature. Dent Traumatol. 2010;26(1):94-97.

6. Sule C, Bilal O. Riga-Fede disease in the upper jaw in an infant. Dermatologic Therapy. [Internet]. 2017 [citado el 20 de octubre de 2017]; e12517. Disponible en https://doi.org/10.1111/dth.12517.
7. Slayton R. Treatment alternatives for sublingual traumatic ulceration (Riga-Fede disease). Pediatr Dent. 2000;22(5):413-414.

8. Yuvaraj M, Kumar R, Aparna R, Radhakrishnan V. Neonatal sublingual traumatic ulceration - case report \& review of the literatura. Dent Traumatol. 2010;26(1):490-495.

9. Elzay R. Traumatic ulcerative granuloma with stromal eosinophilia (Riga-Fede's disease and traumatic eosinophilic granuloma). Oral Surg. 1983;55(4):497506.

10. Goho C. Neonatal sublingual traumatic ulceration (Riga-Fede disease): reports of cases. ASDC J Dent Child. 1996;63(5):362-64.

11. Ziad D. Riga-Fede disease: report of a case and review. J Clin Pediatr Dent. 2001;25(3):209-213.

12. Dunlop R, Barton D, Jones J. Riga-Fede disease: a case report. J Pediatr Health Care. 2013;27(2):155-7.

13. Hardnt E, Weywer H. Odontología infantil. 1Ed. Buenos Aires: Editorial Mundi; 1969.

14. Ziad D. Riga-Fede disease: association with microcephaly. Int J Paediatr Dent. 2002;12(1):442-445.

15. Robson FC, Farli AC, Dione DT, Wanda TG. Natal and neonatal teeth: review of the literature. Pediatr Dent. 2001;21(3):158-162.

16. Rao RS, Mathad SV. Natal teeth: case report and review of literature. J Oral MaxilloFac Pathol. 2009;13(1):41-6.

17. Sung CC, Jae HP, Young CC, Gyu-Tae K. Sublingual traumatic ulceration (a Riga-Fede disease): report of two cases. Dent Traumatol. 2009;25(1):48-50.

18. Erik VDM, Tjalling WV, Henk FE, Jan GV. Traumatic lingual ulceration in a newborn: Riga-Fede disease. Ital J Pediatr. 2012;34(6):8-20.

19. Li J, Zhang Y, Wang N, Bhandari R, Liu Q. Riga-Fede disease in a child. Clin Exp Dermatol. 2016;41(1):285286.

20. Bilal O. Persistent Untreated Riga-Fede Disease for 6 years. Pediatr Dermatol. 2015;32(3):134-135.

21. Costacurta M, Maturo P, Docimo R. Riga-fede disease and neonatal teeth. ORAL \& Implantology. 2012;5(1):26-30.

22. Tandale S, Dave NM, Garasia M. Methemoglobinemia: What the anaesthetist must know. Indian J Anaesth. 2013;57(3):427-8.

23. Guay J. Methemoglobinemia related to local anesthetics: a summary of 242 episodes. Anesth Analg. 2009;108(3):837-845.

24. Veltri K, Rudnick E. Benzocaine-Induced Methemoglobinemia: A Case Report. Pharmacovigilance Forum. 2016;41(3):180-184.

25. Schroeder TH, Dieterich HJ, Mühlbauer B. Methemoglobinemia after axillary block with bupivacaine and additional injection of lidocaine in the operative field. Acta Anaesthesiol Scand. 1999;43(4):480-482. 